\title{
MiCROAMBIENTE LUMÍNICO Y MORFOLOGÍA Y FISIOLOGÍA FOLIAR DE BROMELIA KARATAS (BROMELIACEAE) EN UNA SELVA BAJA CADUCIFOlia de YuCATÁN, MÉXICO
}

\author{
Claudia González-Salvatierra ${ }^{1,2}$, José Luis Andrade ${ }^{1,3}$, Roger Orellana ${ }^{1}$, \\ Luis Manuel Peña-Rodríguez ${ }^{1}$ y Casandra Reyes-García ${ }^{1}$ \\ ${ }^{1}$ Centro de Investigación Científica de Yucatán, Mérida, Yucatán, México \\ ${ }^{2}$ Instituto Potosino de Investigación Científica y Tecnológica, San Luis Potosí, San Luis Potosí, México \\ ${ }^{3}$ Autor para la correspondencia: andrade@cicy.mx
}

\begin{abstract}
Resumen: El metabolismo ácido de las crasuláceas es una adaptación de algunas plantas a condiciones de escasez de agua o bióxido de carbono y está relacionado con mecanismos de respuesta anatómicos, morfológicos y fisiológicos para tolerar períodos prolongados de sequía. La luz puede afectar diferentes procesos metabólicos, fisiológicos y estructurales de las plantas cuando es excesiva, por lo que en ambientes cambiantes, como en las selvas caducifolias, las hojas presentan respuestas de fotoprotección. El objetivo de este trabajo fue determinar esas respuestas, en dos microambientes de luz y dos temporadas del año, en una bromeliácea terrestre (Bromelia karatas) de la selva baja caducifolia en el Parque Nacional Dzibilchaltún, Yucatán. Las características morfológicas y fisiológicas de las hojas reflejaron la influencia del ambiente, lo que les permitió conservar potenciales hídricos diurnos elevados y minimizar la pérdida de agua para mantener la fotosíntesis. Estas características, en conjunto con las espinas foliares y el crecimiento asexual de esta especie, pueden ayudar a explicar su alto valor de importancia ecológica en esta selva baja caducifolia.
\end{abstract}

Palabras clave: fluorescencia de la clorofila, fotoprotección, metabolismo ácido de las crasuláceas, potencial hídrico.

\begin{abstract}
Crassulacean acid metabolism is an adaptation of certain plants to the lack of water or carbon dioxide and it is related to anatomical, morphological, and physiological response mechanisms to cope with long drought periods. High light can affect metabolic, physiological, and structural processes in plants and, in changing environments (such as those of a seasonally dry forests), plant leaves show anatomical and physiological photoprotection responses. The objective of this study was to determine those responses in two light microenvironments and in two seasons for a terrestrial bromeliad (Bromelia karata) from the tropical dry deciduous forest of Dzibilchaltún National Park in Yucatán, Mexico. The morphological characteristics of the leaves of $B$. karatas reflected the influence of the environmental factors and allowed maintaining high diurnal water potentials and diminishing water loss to maintain photosynthesis. These foliar characteristics, along with the foliar spines and asexual growth of this species, can help to explain its high ecological importance value in this tropical dry deciduous forest.
\end{abstract}

Key words: chlorophyll, fluorescence, crassulacean acid metabolism, photoprotection, water potential.

$\mathbf{L}$ a asimilación de $\mathrm{CO}_{2}$ durante la noche, típica de plantas con metabolismo ácido de las crasuláceas (CAM, siglas en inglés), es un mecanismo efectivo de concentración de carbono que además contribuye a la fotoprotección de las plantas expuestas a altas intensidades de luz (Maxwell et al., 1994, 1995). La fotosíntesis CAM es ventajosa en sitios secos porque la fijación de $\mathrm{CO}_{2}$ diurna por la ribulosa-1,5bifosfato carboxilasa/oxigenasa sucede cuando los estomas están cerrados, por lo que el mantenimiento del transporte de electrones previene el daño a los fotosistemas (Niewiadomska y Borland, 2008). La fotoprotección involucra mecanismos que previenen que el exceso de energía lumínica 
absorbida dañe las células fotosintéticas (Logan et al., 1996; Griffiths y Maxwell, 1999). En la mayoría de los casos, las respuestas de fotoprotección son rápidas, como los aumentos en las concentraciones foliares de carotenoides y $\alpha$-tocoferol pocas horas después de exposición a alta intensidad de luz (Matsubara et al., 2009).

En sitios con cambios en la disponibilidad de agua, las plantas han desarrollado ciertas características xerofíticas para mantener el agua en sus tejidos, como son baja transpiración cuticular, reducción en la densidad estomática $(\mathrm{Bu}-$ ttery et al., 1993), presencia de tricomas reflejantes (Van Rensburg et al., 1994), pérdida de hojas o reducción de sus dimensiones, o bien tienen la capacidad de responder a diferentes niveles de exposición a la luz con cambios en la estructura interna del tejido fotosintético, como cantidad y distribución del parénquima en empalizada y esponjoso, así como de su estructura externa (Givnish, 1988; Parlevliet et al., 1991; Osborne et al., 1994; Nobel, 1995; Anderson et al., 1997; Niyogi, 2000; Larcher, 2003; Peñuelas y MunnéBosch, 2005; Tanaka et al., 2008). Los cambios estructurales del tejido fotosintético en respuesta a la luz influyen directamente en la captación de $\mathrm{CO}_{2}$ porque cambian la resistencia difusiva del $\mathrm{CO}_{2}$, lo que repercute en la eficiencia fotosintética y en la eficiencia de uso del agua (Patton y Jones, 1989; Parkhurst y Mott, 1990; Vogelman, et al., 1996; Nobel y de la Barrera, 2004).

La familia Bromeliaceae tiene una amplia distribución en un extenso intervalo de condiciones ambientales y presenta una gran variación morfo-anatómica y de formas de vida, con especies adaptadas a sitios totalmente expuestos a la luz y otras adaptadas a la sombra (Martin, 1994; Skillman y Winter, 1997; Benzing, 2000). En las selvas bajas caducifolias de Yucatán coexisten varias plantas CAM, terrestres y epifitas, adaptadas a los cambios de la intensidad de luz y disponibilidad de agua durante el año, que muestran cambios en la fotosíntesis y en la producción de metabolitos foliares en respuesta al ambiente lumínico (González-Salvatierra et al., 2010; Ricalde et al., 2010). Bromelia karatas L. es una especie terrestre con un valor alto de importancia ecológica entre las plantas terrestres perennes de algunas selvas bajas caducifolias del norte de la Península de Yucatán, principalmente por su abundancia y cobertura (Ricalde et al., 2010). Por esto, el objetivo de este trabajo fue determinar los cambios fisiológicos, anatómicos y morfológicos en las hojas de esta especie CAM, en dos condiciones de exposición a la luz, durante la estación seca y la estación de lluvias, en una selva baja caducifolia.

\section{Materiales y métodos}

Especie de estudio. Bromelia karatas se distribuye en varias selvas secas de la Península de Yucatán y se ha reportado su existencia desde Sinaloa hasta Ecuador y Brasil (Ramírez et al., 2004). Es una bromeliácea terrestre de gran tamaño ( 1.5 a 3 m de porte) que forma colonias de dos o tres rosetas; las hojas cuentan con espinas marginales y con tricomas foliares hidrofóbicos (Pittendrigh, 1948; Benzing, 2000; Pierce et al., 2001). De acuerdo con Benzing (2000), $B$. karatas pertenece a la clasificación de tipo ecológico $\mathrm{V}$ y es una especie CAM obligada (Martin, 1994; González-Salvatierra et al., 2010). Se eligió una población de B. karatas que consistía de 34 individuos en el sitio de estudio, varios individuos crecían bajo los árboles y otros en sitios expuestos.

Sitio de estudio. El trabajo de campo se llevó a cabo en el Parque Nacional Dzibilchaltún $\left(21^{\circ} 05^{\prime} \mathrm{N}, 89^{\circ} 99^{\prime} \mathrm{O}, 10 \mathrm{~m}\right.$ s.n.m.) en el estado de Yucatán, México; con una precipitación media anual de $700 \mathrm{~mm}$ y una temperatura promedio de $25.8^{\circ} \mathrm{C}$. La temporada de lluvias es de junio a noviembre y la seca de diciembre a mayo (Orellana et al., 1999). Esta última se caracteriza porque la mayoría de los árboles pierden sus hojas (c.a. 70\%, Mondragón et al., 2004; ValdezHernández et al., 2010).

Mediciones ambientales. Las variables ambientales se obtuvieron con una estación meteorológica instalada en el Parque Nacional Dzibilchaltún, durante un periodo dentro de la temporada de lluvias (agosto-septiembre 2006) y de sequía (abril-mayo 2007). Las variables que se midieron fueron el flujo fotónico fotosintético (FFF; $\mu \mathrm{mol} \mathrm{m}^{-2} \mathrm{~s}^{-1}$ ) con un sensor cuántico (LI-190SB, Li-Cor, Inc., Lincoln, Nebraska, EUA), la precipitación pluvial con un pluviómetro (TR 525M, Texas Electronics, Inc., Dallas, Texas, EUA) y la temperatura del aire y la humedad relativa con una sonda Vaisala (HMP35C-L, Campbell Scientific, Logan, Utah, EUA). Todas las variables se registraron cada $15 \mathrm{~s}$ y sus valores promedios se almacenaron cada $10 \mathrm{~min}$ con un sistema de adquisición de datos (CR21X, Campbell Scientific). El déficit de presión de vapor se determinó a partir de los valores de temperatura y humedad relativa (Jones, 1992; Andrade, 2003).

Microambiente lumínico. Se determinó la cantidad y el porcentaje de FFF incidente sobre los individuos de Bromelia karatas con un sensor cuántico (LI190SA, LI-COR, Lincoln, Nebraska, EUA) conectado a un medidor (LI250-A, LI-COR) a una altura de aproximadamente $20 \mathrm{~mm}$ sobre cada planta en tres puntos diferentes. En julio 2006, de los 34 individuos de la población seleccionada se escogieron al azar 3 - 6 plantas sombreadas (19 plantas recibían $20 \%$ del FFF total diario sobre el dosel de la selva) y 3 - 6 expuestas (15 plantas recibían $\sim 70 \%$ del FFF) para las mediciones fisiológicas, morfológicas y anatómicas.

Variables fisiológicas.

Potencial hídrico.-Se recolectaron muestras de hojas (hojas totalmente expandidas de la parte media de la planta) de plantas expuestas y bajo sombra $(n=3-6$ plantas $)$ en dos 
tiempos durante el día: pre-alba (entre las 05:30 y 06:00 h) y mediodía, durante la temporada de lluvias y de sequía. Las muestras se almacenaron en bolsas plásticas sobre hielo a $4{ }^{\circ} \mathrm{C}$, se transportaron al laboratorio y se midió el potencial hídrico foliar $\left(\Psi_{\mathrm{f}}\right)$ con la técnica de punto de rocío (WP4, Decagon, Devices, Inc. Washington, EUA). Las muestras de hojas se cortaron en piezas cuadradas para cubrir el área de la cámara del instrumento. La cutícula foliar se rayó ligeramente con una navaja como sugiere el manual del equipo.

Acidez en el tejido.-Para caracterizar la actividad CAM, se recolectaron muestras de hojas de plantas expuestas y de sombra ( $\mathrm{n}=5$ plantas) durante las temporadas de lluvias y de sequía, al atardecer y a pre-alba del día siguiente. Las muestras de hoja se obtuvieron con un sacabocados (1.54 $\mathrm{cm}^{2}$ ), se transportaron y conservaron en $1.5 \mathrm{ml}$ de etanol al $70 \%$ (aproximadamente $2 \mathrm{~h}$ ) y la extracción del contenido celular se realizó por ebullición (para eliminar el etanol) en $10 \mathrm{ml}$ de agua destilada durante $15 \mathrm{~min}$; posteriormente se agregaron $50 \mathrm{ml}$ de agua destilada. El sobrenadante resultante se tituló con $\mathrm{NaOH} 0.005 \mathrm{~N}$ hasta un $\mathrm{pH}$ de 7 con un potenciómetro (Oakton ${ }^{\circledR} \mathrm{pH} 510$ series; Pearcy et al., 1989; Zotz y Andrade, 1998). La acidificación nocturna $\left(\Delta \mathrm{H}^{+}\right)$se estimó a partir de la concentración del ión hidrógeno $\left(\mathrm{H}^{+}\right)$ pre-alba menos la $\mathrm{H}^{+}$al atardecer.

Concentración de pigmentos.-Durante cada temporada, se recolectaron de cinco a seis hojas de tres plantas expuestas y tres bajo sombra (a las 08:00 h), se mantuvieron a $4{ }^{\circ} \mathrm{C}$ y se transportaron al laboratorio. Los pigmentos se extrajeron de acuerdo al procedimiento de Hendry y Price (1993) en la obscuridad: se cortaron $50 \mathrm{mg}$ de muestra de hojas (peso fresco) y se molieron con nitrógeno líquido en un mortero frío, se colocaron $2 \mathrm{ml}$ de acetona al $80 \%$, la extracción se colocó en un tubo Eppendorf de $2 \mathrm{ml}$ y las muestras se centrifugaron a 10,000 durante $5 \mathrm{~min}$. El sobrenandante se recuperó en un tubo de ensaye y se aforó con $5 \mathrm{ml}$ de acetona al $80 \%$, posteriormente se midió la absorbancia con un espectrofotómetro (Beckman Coulter DU650); las lecturas se aplicaron a $645 \mathrm{~nm}$ y $663 \mathrm{~nm}$ para clorofilas y a $470 \mathrm{~nm}$ para carotenoides totales. La concentración de los pigmentos se calculó de acuerdo a Hendry y Price (1993) para clorofilas y Wellburn (1994) para carotenoides.

Fluorescencia de la clorofila.- Las mediciones se realizaron en hojas totalmente expandidas de la parte media de la roseta. Para estimar el rendimiento cuántico máximo $\left(\mathrm{F}_{\mathrm{v}} / \mathrm{F}_{\mathrm{m}}\right.$; fluorescencia variable/fluorescencia máxima) las mediciones se efectuaron pre-alba. Asimismo, se midieron parámetros relacionados (por sus siglas en inglés: la disipación no fotoquímica, NPQ; el rendimiento cuántico del fotosistema II, ФPSII; y la tasa de transporte de electrones, ETR) con un medidor de fluorescencia portátil (Mini-PAM; H. Walz, Effeltrich, Alemania). Las mediciones se ejecutaron durante las temporadas de lluvias y de sequía en seis individuos de plantas expuestas y seis de plantas en sombra. Las mediciones de la ETR, el NPQ, la ФPSII y el FFF se realizaron du- rante el curso de un día, en intervalos de $3 \mathrm{~h}$ (05.00, 08.00, $11.00,14.00$ y $17.00 \mathrm{~h}$ ).

Las curvas de respuesta a la luz se ejecutaron a las 11:00 h en plantas expuestas y bajo sombra para determinar el punto de saturación de sus hojas durante la temporada lluviosa y seca (Rascher et al., 2000). Las plantas se aclimataron a la oscuridad cubriéndolas con una bolsa negra opaca por 20 min. Se aplicaron nueve pulsos de luz actínica hasta los $1,400 \mu \mathrm{mol} \mathrm{m} \mathrm{m}^{-2} \mathrm{~s}^{-1}$, el primer pulso refleja el rendimiento en ausencia de luz actínica y los ocho pulsos de luz consecutivos restantes se requieren para determinar el punto de saturación a la luz. La ETR (la cual refleja la eficiencia con la cual la energía de excitación capturada por los pigmentos antena es transferida hacia los aceptores de electrones en los centros de reacción del PSII; Johnson et al., 1993; Demming-Adams et al., 1995; Maxwell y Johnson, 2000) se calculó como $\Phi$ PSII $\times$ FFF $\times 0.5 \times 0.84$, donde el factor 0.5 indica que la energía de excitación se distribuye igualmente entre los dos fotosistemas y el factor 0.84 corresponde a la fracción de luz absorbida y utilizada por el PSII (Ritchie y Bunthawin, 2010). Este factor fue recientemente validado para otras dos especies de bromeliáceas (Stemke y Santiago, 2011). El punto de saturación a la luz se calculó como el valor de FFF cuando se alcanzó el 90\% de la ETR máxima.

Material vegetal para microscopía. Para determinar los caracteres morfológicos de Bromelia karatas se recolectaron 20 hojas maduras de plantas expuestas y bajo sombra, durante la temporada de sequía. Para el análisis en el microscopio electrónico de barrido las hojas fueron conservadas en alcohol glicerinado (alcohol:agua:glicerina 70:29:1), para su análisis posterior.

Técnica para observar las células de mesofilo. Las hojas recolectadas en fresco se lavaron y se hicieron cortes finos con ayuda de una navaja, aproximadamente a un grosor de $10 \mu \mathrm{m}$ y fueron colocados en un portaobjetos. Los cortes se fijaron de manera semipermanente con alcohol polivinílico y selladas con esmalte de uñas. Las observaciones al microscopio óptico (Leica EDM) se realizaron utilizando los aumentos $10 \mathrm{X}$ y $40 \mathrm{X}$ y se tomaron fotografías para diferenciar las capas de células del mesofilo (parénquima en empalizada y parénquima esponjoso), así como para visualizar el tamaño y forma de los canales de aire y la distribución y tipo de los haces vasculares.

Microscopía electrónica de barrido. Para la descripción de los tricomas y los estomas se hicieron cortes de $5 \times 5 \mathrm{~mm}^{2}$ en la parte central de las hojas y las observaciones se realizaron con ayuda de un microscopio electrónico de barrido (MEB; Jeol, JSM-6360LV). Las muestras fueron deshidratadas utilizando soluciones con diferentes concentraciones de etanol (desde 10\% hasta 100\%) durante $24 \mathrm{~h}$. Las muestras deshidratadas se secaron a punto crítico con $\mathrm{CO}_{2}$ (SAMDRI 795, 
semiautomática) para sustituir el etanol por $\mathrm{CO}_{2}$ y evitar con esto la deformación de los tejidos. Posteriormente, se realizó la fijación de las muestras en portaobjetos metálicos de cobre y se cubrieron con una capa de oro-paladio utilizando una metalizadora (Denton Vacuum Desk II a $15 \mathrm{kV}$ ).

Análisis estadísticos. Un análisis de varianza de dos vías (factores temporada y nivel de luz) se realizó para las variables flujo de fotones para la fotosíntesis, potencial hídrico, acidez del tejido, concentración de pigmentos fotosintéticos y las variables de fluorescencia de la clorofila. $\mathrm{La}$ comparación de medias para las variables con diferencias significativas se efectuó con la prueba de Tukey. Las diferencias entre temporadas para las variables ambientales se evaluaron con una prueba t de Student. Todas las pruebas fueron realizadas con el paquete estadístico STATISTICA 7.0 (Statsoft, EUA).

\section{Resultados}

Mediciones ambientales y microambiente lumínico. Durante la temporada seca (abril-mayo 2007), el flujo fotónico fotosintético (FFF) diario promedio por encima del dosel fue significativamente mayor $\left(48.4 \mathrm{~mol} \mathrm{~m}^{-2} \mathrm{~d}^{-1}\right)$ que durante la temporada de lluvias (agosto-septiembre 2006; $45.8 \mathrm{~mol}$ $\mathrm{m}^{-2} \mathrm{~d}^{-1} ; P<0.05$; Cuadro 1). Por otro lado, en la temporada lluviosa se observó una ligera disminución no significativa del déficit de presión de vapor máximo con respecto al de la temporada seca $(P>0.05$; Cuadro 1$)$.

En la temporada de lluvias, el porcentaje del FFF diario total recibido sobre las plantas bajo sombra fue de $16 \%$ del recibido encima del dosel, en comparación con las plantas expuestas que recibieron el 70\% FFF diario total; en la temporada seca, el FFF total recibido sobre las plantas se incrementó a 54\% en las plantas sombreadas y a $90 \%$ en las expuestas (Cuadro 2). El FFF total diario fue significativamente diferente entre temporadas y microambientes lumínicos $(P<0.05)$. Las plantas bajo sombra recibieron un promedio de 3.4 veces más FFF durante la temporada seca que durante la temporada de lluvias $(P<0.05$; Cuadro 2$)$.

Cuadro 1. Promedio del flujo fotónico fotosintético (FFF) diario encima del dosel $( \pm \mathrm{EE}$ ), déficit de presión de vapor (DPV) máximo y mínimo, y precipitación mensual en la selva baja caducifolia de Dzibilchaltún, Yucatán, México, en la época lluviosa (agosto-septiembre 2006) y seca (abril-mayo 2007).

\begin{tabular}{lcccc}
\hline & $\begin{array}{c}\mathrm{FFF} \\
(\mathrm{mol} \mathrm{m}-2 \mathrm{~d}-1)\end{array}$ & $\begin{array}{c}\mathrm{DPV}_{\text {max }} \\
(\mathrm{kPa})\end{array}$ & $\begin{array}{c}\mathrm{DPV}_{\text {min }} \\
(\mathrm{kPa})\end{array}$ & $\begin{array}{c}\text { Precipitación } \\
(\mathrm{mm})\end{array}$ \\
\hline Agosto & $44.55 \pm 1.5$ & 3.44 & 1.54 & 270.19 \\
Septiembre & $47.09 \pm 0.9$ & 5.73 & 0.55 & 82.89 \\
Abril & $47.29 \pm 1.4$ & 5.24 & 1.14 & 0.2 \\
Mayo & $49.47 \pm 1.4$ & 5.48 & 1.81 & 27.09 \\
\hline
\end{tabular}

Cuadro 2. Promedio del flujo fotónico fotosintético (FFF) diario, concentración de clorofila y carotenoides, y eficiencia fotosintética máxima del fotosistema II $\left(F_{\sqrt{ }} \mathrm{F}_{\mathrm{m}}\right)$ en plantas expuestas y bajo sombra de Bromelia karatas, durante la temporada de lluvias (septiembre 2006) y de sequía (abril 2007) en la selva baja caducifolia de Dzibilchaltún, Yucatán, México. Datos son medias \pm EE $(n=5)$.

\begin{tabular}{|c|c|c|c|c|}
\hline & \multicolumn{2}{|c|}{ Expuestas } & \multicolumn{2}{|c|}{ Sombreadas } \\
\hline & $\begin{array}{l}\text { Temporada } \\
\text { de lluvia }\end{array}$ & $\begin{array}{l}\text { Temporada } \\
\text { de sequía }\end{array}$ & $\begin{array}{l}\text { Temporada } \\
\text { de lluvia }\end{array}$ & $\begin{array}{l}\text { Temporada } \\
\text { de sequía }\end{array}$ \\
\hline $\begin{array}{l}\text { FFF } \\
\left(\mathrm{mol} \mathrm{m}^{-2} \mathrm{~d}^{-1}\right)\end{array}$ & $30.85^{\mathrm{a}} \pm 2.8$ & $37.59^{\mathrm{a}} \pm 1.2$ & $7.62^{c} \pm 0.8$ & $23.02^{b} \pm 1.9$ \\
\hline $\begin{array}{l}\text { Clorofila total } \\
\left(\mu g g^{-1} p f\right)\end{array}$ & $315.89^{b} \pm 33.4$ & $140.47^{c} \pm 39.5$ & $634.99^{\mathrm{a}} \pm 49.7$ & $300.41^{b} \pm 34.4$ \\
\hline $\begin{array}{l}\text { Carotenoides } \\
\left(\mu \mathrm{g} \mathrm{g}^{-1} \mathrm{pf}\right)\end{array}$ & $33.84^{\mathrm{c}} \pm 4.9$ & $54.35^{b} \pm 10.8$ & $38.91^{b c} \pm 3.4$ & $100.74^{a} \pm 6.4$ \\
\hline $\mathrm{F} / \mathrm{F}_{\mathrm{m}}$ & $0.75^{\mathrm{ab}} \pm 0.02$ & $0.65^{b} \pm 0.04$ & $0.82^{\mathrm{a}} \pm 0.01$ & $0.71^{\mathrm{ab}} \pm 0.04$ \\
\hline
\end{tabular}

Letras diferentes indican diferencias significativas entre estaciones y exposición, la diferencia entre medias fue determinada por una prueba de Tukey $(P<0.05)$.

Potencial hídrico y acidez, titulable. En la temporada de lluvias, los valores más negativos de potencial hídrico foliar $\left(\Psi_{\mathrm{f}}\right)$ se registraron al mediodía en todas las plantas, independientemente de su exposición al sol, y fueron significativamente menores al $\Psi_{\mathrm{f}}$ pre-alba $(P<0.004$; Figura 1$)$. En la temporada seca, no hubo diferencias significativas entre $\Psi_{\mathrm{f}}$ al mediodía y pre-alba en plantas sombreadas y expuestas (Figura 1).

La hojas de Bromelia karatas presentaron valores altos de acidificación nocturna $\left(\Delta \mathrm{H}^{+}\right.$, mayor de $300 \mathrm{mmol} \mathrm{H}^{+} \mathrm{m}^{-2}$

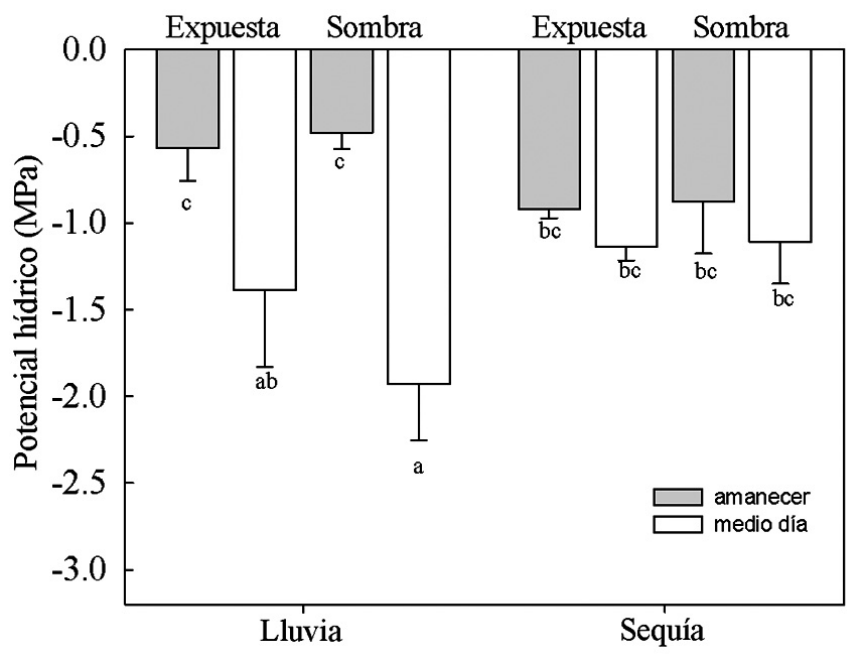

Figura 1. Potencial hídrico foliar pre-alba y al medio día de plantas expuestas y bajo sombra de Bromelia karatas durante la temporada lluviosa y seca en la selva baja caducifolia de Dzibilchaltún, Yucatán, México. Los datos son medias \pm EE $(n=3-6)$. Letras diferentes indican diferencias significativas calculadas por una prueba de Tukey $(P<0.05)$. 
para plantas expuestas durante la temporada de lluvia; Figura 2). Se observaron diferencias significativas entre temporadas (Figura 2; $P<0.010$ ); en la temporada de sequía, las hojas de las plantas bajo sombra presentaron los valores de $\Delta \mathrm{H}^{+}$significativamente más bajos que las hojas de las plantas expuestas $(P<0.05)$.

Contenido de pigmentos y parámetros de fluorescencia. Las hojas de las plantas bajo sombra presentaron un mayor contenido de clorofilas que las de las plantas expuestas (Cuadro 2; $P<0.05)$, especialmente durante la temporada de sequía. Las hojas de las plantas bajo sombra presentaron 2.5 veces más contenido de carotenoides durante la temporada de sequía que durante la temporada de lluvia $(P<0.05)$.

En la temporada seca, los valores de la eficiencia cuántica máxima $\left(\mathrm{F}_{\mathrm{v}} / \mathrm{F}_{\mathrm{m}}\right)$ de hojas de plantas expuestas fueron los más bajos registrados (Cuadro 2; $P<0.05$ ). En la temporada de lluvias, las hojas de las plantas expuestas y bajo sombra presentaron valores altos de $\mathrm{F}_{\mathrm{v}} / \mathrm{F}_{\mathrm{m}}$. En ambas temporadas, los valores del rendimiento cuántico del fotosistema II (ФPSII) disminuyeron a valores de $0.3-0.4$ en las hojas de plantas expuestas y en las de plantas sombreadas en la época de sequía (Figura 3E, F). Los valores de la tasa de transporte de electrones (ETR) fueron altos en las hojas de plantas expuestas durante ambas temporadas (Figuras 3I, J). En la temporada de sequía, los valores de ETR se incrementaron al medio día, mientras que los valores de NPQ se mantuvieron bajos, tanto para las hojas de plantas expuestas como las de plantas bajo sombra (Figuras 3G, 3H). Por otro lado, el

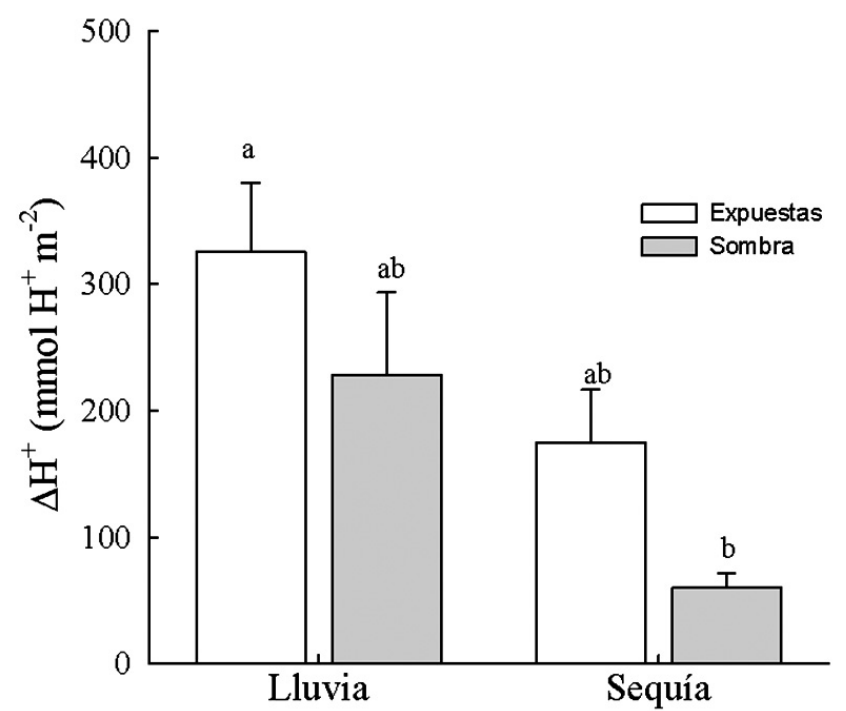

Figura 2. Diferencias de acidez $\left(\Delta \mathrm{H}^{+}\right.$; concentración de $\mathrm{H}^{+}$en el atardecer menos $\mathrm{H}^{+}$pre-alba del día siguiente) de hojas de Bromelia karatas para plantas expuestas (barras blancas) y bajo sombra (barras grises), durante las temporadas de lluvias y de sequía, en la selva baja caducifolia de Dzibilchaltún, Yucatán, México. Los datos son medias $\pm \mathrm{EE}(\mathrm{n}=5)$. Letras diferentes indican diferencias significativas, calculadas por una prueba de Tukey $(P<0.05)$.
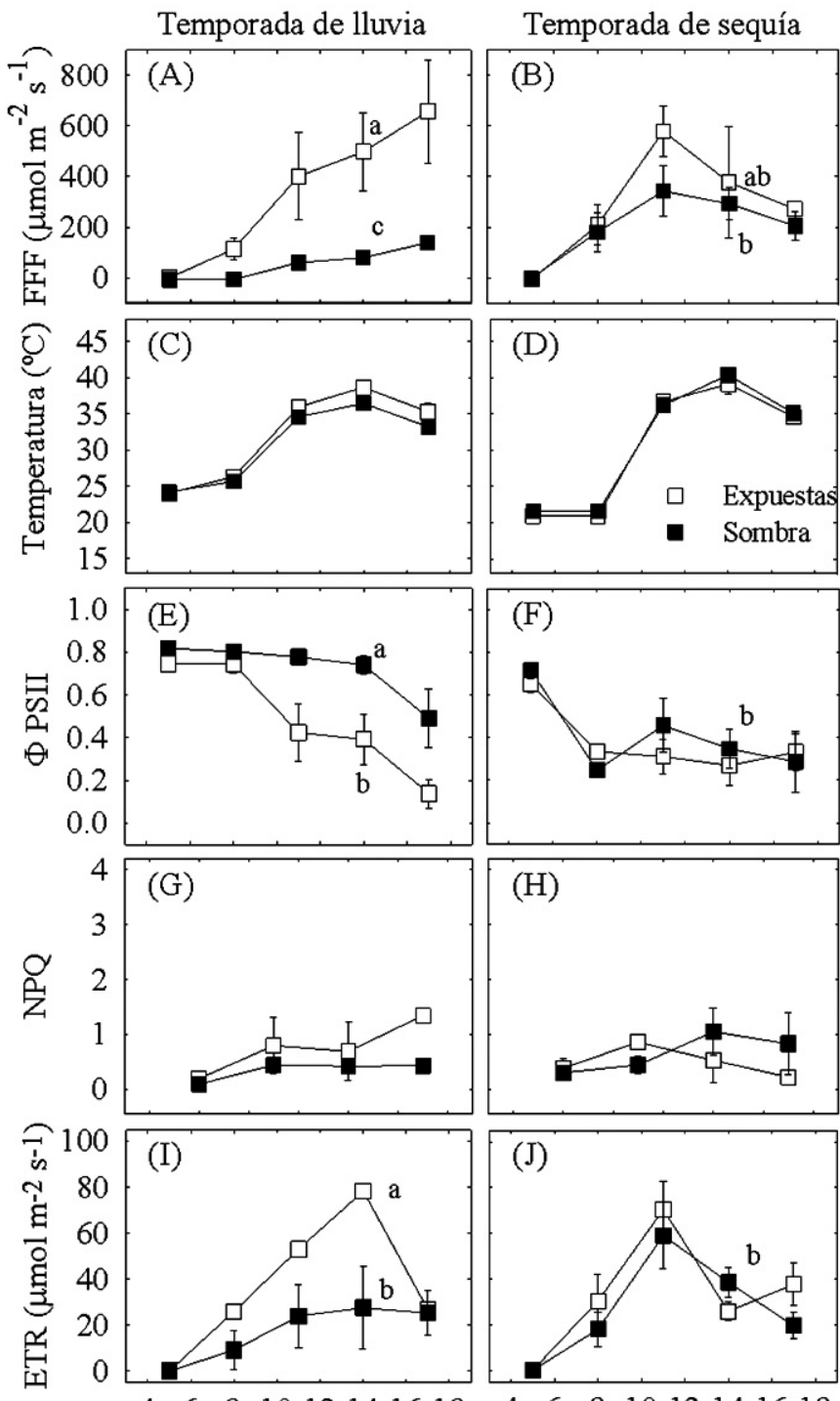

(I)

$(1)$

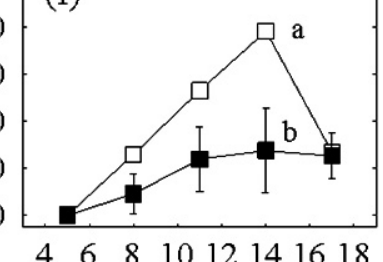

$(\mathrm{J})$

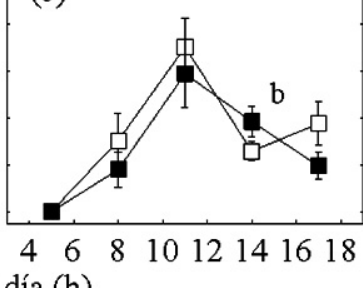

Hora del día (h)

Figura 3. Curso diurno del flujo fotónico fotosintético (FFF; A y B) y temperatura (C y D) y curso diario de los parámetros de la fluorescencia de la clorofila: rendimiento cuántico del fotosistema II (ФPSII; E y F), disipación no fotoquímica (NPQ; G y H) y la tasa de transporte de electrones (ETR; I y J), para hojas de plantas expuestas y bajo sombra de Bromelia karatas durante las temporadas de lluvias y de sequía (paneles de la derecha) en la selva baja caducifolia de Dzibilchaltún, Yucatán, México. Los datos son medias $\pm E E(n=3)$. Diferentes letras indican diferencias significativas entre temporadas, calculadas por una prueba de Tukey $(P<0.05)$.

punto de saturación de luz fue significativamente diferente entre temporadas $(P<0.05)$, pero no se observaron diferencias entre plantas expuestas y bajo sombra. En la temporada de lluvia, el punto de saturación de luz fue alto $(1,200 \mu \mathrm{mol}$ $\left.\mathrm{m}^{-2} \mathrm{~s}^{-1}\right)$; sin embargo, en la temporada de sequía, el punto de saturación de luz se alcanzó a los $535 \mu \mathrm{mol} \mathrm{m} \mathrm{m}^{-2} \mathrm{~s}^{-1}$. 

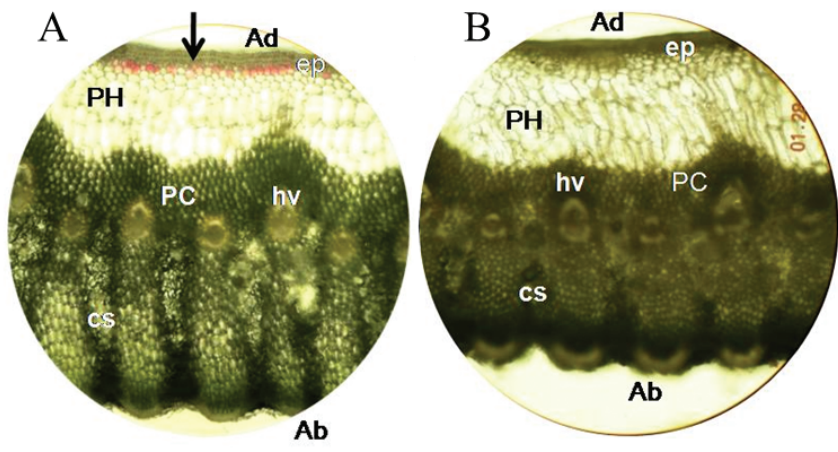

Figura 4. Microfotografías de las secciones transversales de hojas de plantas expuestas y de sombra de Bromelia karatas. A, sección transversal de hoja expuesta (10x). B, sección transversal de hoja de sombra (10x). $\mathrm{PH}$ : parénquima esponjoso; $\mathrm{PC}$ : parénquima en empalizada; hv: haz vascular; ca: canales de aire; Ab: superficie abaxial; Ad: superficie adaxial; ep: epidermis; cs: cavidad subestomática. Las flechas indican la localización de las antocianinas.

Caracteres morfológicos. Bromelia karatas presentó caracteres anatómicos propios de una planta CAM, con células del mesofilo grandes y fuertemente compactadas (Figura 4). En los cortes transversales de las hojas se distinguieron las superficies adaxial y abaxial de las hojas de plantas expuestas y de sombra, las cuales presentaron varias capas de mesofilo compuesto por parénquima en empalizada y esponjoso, así como haces vasculares en la parte media del mesofilo que estuvieron total o parcialmente rodeados por fibras y canales de aire. Se observó una compactación del parénquima en empalizada hacia la superficie abaxial (Figura 4A, B).

Microscopía electrónica de barrido. La superficie adaxial de las hojas de Bromelia karatas no presentó ni tricomas ni estomas (Figura 5A). En la superficie abaxial se distinguieron regiones costales e intercostales (Figura 5B); sobre las regiones intercostales se observaron los tricomas, distribuidos en hileras longitudinales, los que presentaron células del ala y un disco central, el cual fue poco sobresaliente (Figura 5C). Los estomas se encontraron dentro de las regiones intercostales, dispuestos en depresiones (debajo de las células epidérmicas), cubiertos por las alas de los tricomas, y presentaron una distribución continua (Figura 5D).

\section{Discusión}

Las hojas de Bromelia karatas presentaron diferencias diurnas de potencial hídrico $\left(\Psi_{\mathrm{f}}\right)$ en la temporada de lluvias, con valores menores al mediodía en comparación con los valores pre-alba, lo cual podría considerarse inusual para una planta CAM (Griffiths, 1989; Andrade et al., 2009). En plantas CAM, la acumulación de ácidos orgánicos por la noche disminuye el $\Psi_{\mathrm{f}}$ pre-alba, mientras que durante el día el $\Psi_{\mathrm{f}}$ aumenta porque la producción de carbohidratos ocurre con los estomas cerrados (Smith et al., 1986; Griffiths,
1989). Sin embargo, se ha reportado que algunas especies de bromeliáceas epifitas abren los estomas durante el día en condiciones de disponibilidad de agua en el medio y asimilan el $\mathrm{CO}_{2}$ por la vía $\mathrm{C}_{3}$ hasta en un $20 \%$ del total fijado (Graham y Andrade, 2004; Andrade et al., 2009). La transpiración diurna de las hojas de B. karatas provocaría entonces una disminución en los valores del $\Psi_{\mathrm{f}}$ del mediodía. Los valores similares de $\Psi_{\mathrm{f}}$ pre-alba en las dos temporadas resaltan la capacidad de esta especie para acumular agua en los tejidos foliares o en la raíz. El déficit de presión de vapor máximo no fue diferente en ambas épocas de medición, por lo que no debe tener influencia en la desecación de los tejidos foliares, pero los valores del déficit de presión de vapor mínimo si podría permitir la supervivencia de los individuos jóvenes aislados, porque en estas selvas se produce la deposición de rocío durante casi todo el año (Andrade, 2003). Además, las raíces de esta especie podrían acceder a otras fuentes de agua proporcionadas por los árboles, relacionado con el fenómeno de redistribución hidráulica, como se ha documentado para la especie desértica Yucca schidigera (Yoder y Nowak, 1999). Reyes-García y Griffiths (2009) sugieren la necesidad de estudiar los cambios estacionales en las raíces de $B$. karatas, y analizar el efecto de los pulsos de lluvia durante la temporada de sequía sobre la activación de las raíces.

Como muchas plantas CAM, Bromelia karatas tiene adaptaciones para evitar los daños fisiológicos causados por la sequía mediante la presencia de caracteres anatómicos y morfológicos como cutículas impermeables, baja frecuencia de estomas, un parénquima esponjoso bien desarrollado, con

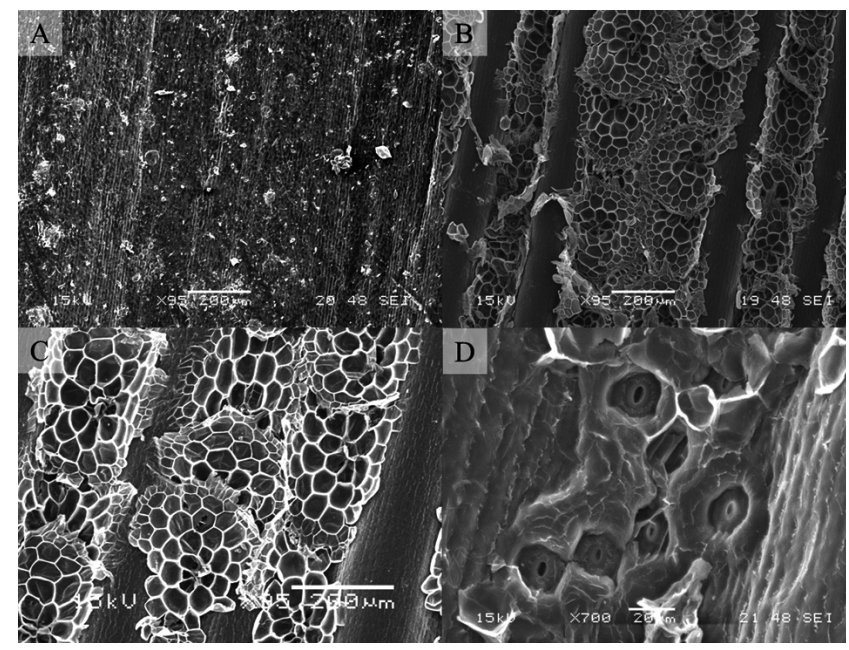

Figura 5. Microfotografías del microscopio electrónico de barrido (MEB) de las hojas de Bromelia karatas. A, vista general de la superficie adaxial sin tricomas y sin estomas; B, tricomas agrupados en hileras longitudinales, sobre las regiones intercostales; C, detalle de los tricomas protegiendo los estomas; D, estomas hundidos dentro de las regiones intercostales, por debajo de la epidermis. $\mathrm{La}$ línea blanca representa la escala. 
vacuolas grandes, lo que les confiere suculencia a las hojas y cuya función es almacenar agua y ácidos orgánicos. Además, a lo largo del mesofilo, dentro del parénquima en empalizada se observaron canales de aire, que tienen la función de transportar agua por capilaridad desde la raíz hacía el parénquima esponjoso, como un mecanismo eficiente de transporte de agua (Smirnoff y Crawford, 1983; Segecin y Scatena, 2004; Scatena y Segecin, 2005). Los canales de aire se encuentran conectados a las cámaras subestomáticas que, además de conferir una mayor flexibilidad, permiten una mayor circulación de gases en el interior de las hojas (Mauseth, 1988). Por otro lado, las hojas de B. karatas cuentan con los estomas pequeños y numerosos, que se encuentran hundidos dentro de canales intercostales de la superficie abaxial y protegidos por los tricomas, lo que les permite disminuir la pérdida de agua por transpiración. Con estas características de ahorro de agua, $B$. karatas puede mantener su $\Psi_{\mathrm{f}}$ por encima de los -2.0 MPa durante la temporada de sequía, y así mantener la apertura nocturna de estomas y la continuidad de la fotosíntesis a bajo $\Psi$ en el suelo (Goldstein et al., 1991; Tyree et al., 2002; Lüttge, 2004; Pimienta-Barrios et al., 2006).

El contenido de ácidos orgánicos, el cual refleja de manera indirecta la asimilación de $\mathrm{CO}_{2}$ y la intensidad del CAM, fue más alto en las hojas de plantas expuestas que en las de plantas de sombra durante ambas temporadas; los valores son similares a los encontrados en otras bromeliáceas terrestres adaptadas a sitios expuestos (Scarano et al., 2002; Ricalde et al., 2010). Así, las reservas de $\mathrm{CO}_{2}$ en los tejidos del parénquima en empalizada permiten el mantenimiento diurno del transporte de electrones (Griffiths et al., 1986; Maxwell et al., 1995).

Las hojas de Bromelia karatas presentaron respuestas típicas de aclimatación a sol y sombra, con mayores concentraciones de clorofila total en plantas de sombra comparadas con plantas expuestas (Givnish, 1988; Martin et al., 1999). Los valores de clorofilas totales fueron similares y comparables con los encontrados en otras bromeliáceas terrestres (Fetene et al., 1990; Benzing, 2000; Endres y Mercier, 2001; Skillman et al., 2005; Matsubara et al., 2009). Durante la temporada de sequía, como respuesta al exceso de luz, los tejidos de las hojas de plantas expuestas y de sombra de $B$. karatas disminuyeron el contenido de clorofilas e incrementaron el contenido de carotenoides. En las hojas expuestas de algunas bromeliáceas terrestres, como Aechmea magdalenae y $B$. humilis, se incrementa el contenido de pigmentos del ciclo de las xantofilas, lo que reduce el daño oxidativo por saturación de luz y que además está relacionado con la disipación del exceso de energía (Königer et al., 1995; Skillman y Winter, 1997; Müller et al., 2001; Havaux et al., 2007; Matsubara et al., 2009). En diferentes grupos de plantas aclimatadas a alta intensidad lumínica, la tendencia general es el decremento de la actividad fotosintética, en donde una disminución en los valores de la eficiencia fotosintética del fotosistema II (ФPSII) probablemente se deba a la disipación de calor (Demmig-Adams et al., 1995). En las hojas de $B$. karatas, los valores de la eficiencia fotosintética máxima $\left(\mathrm{F}_{\mathrm{v}} / \mathrm{F}_{\mathrm{m}}\right.$; entre 0.7 y 0.8$)$ sugieren que los centros funcionales del PSII no se encuentran dañados y por lo tanto no ocurre fotoinhibición. Lo mismo sucede en la bromeliácea terrestre A. magdalenae que presenta también valores altos de $\mathrm{F}_{\mathrm{v}} / \mathrm{F}_{\mathrm{m}}$ en sus hojas aún en condiciones de alta intensidad lumínica (Pfitsch y Smith, 1988; Königer et al., 1995; Skillman y Winter, 1997). Adicionalmente, los tejidos foliares de las hojas expuestas de $B$. karatas mantienen una alta tasa de transporte de electrones (ETR), que se correlaciona con el aumento del contenido foliar de compuestos con actividad antioxidante, especialmente en la época seca (González-Salvatierra et al., 2010).

Las hojas de Bromelia karatas presentan importantes caracteres morfo-anatómicos de adaptación a la alta intensidad de luz, lo que incluye un mesofilo diferenciado en parénquima esponjoso y parénquima en empalizada. El parénquima esponjoso tiene la función de almacenar el agua, filtrar la luz y mantener hidratado al parénquima en empalizada durante la temporada de sequía (Sajo et al., 1998; Zotz y Hietz, 2001; Proença y Sajo, 2007). El parénquima en empalizada presenta células bien diferenciadas con una alta densidad de cloroplastos. Se ha reportado que una diferenciación y distribución del parénquima en empalizada similar a la observada en $B$. karatas, aunado a la presencia de carotenoides y otros pigmentos no fotosintéticos, pueden modular de manera significativa la captura de luz (Tardy et al., 1998). Adicionalmente, para contrarrestar los efectos de la alta intensidad de luz durante la sequía, las concentraciones foliares de antocianinas en B. karatas aumentan a casi el triple (González-Salvatierra et al., 2010).

En sitios expuestos, las hojas de esta especie generalmente producen más ácidos orgánicos durante todo el año que las hojas de plantas bajo sombra, pero en comunidades con mayor precipitación, la acidez tisular puede ser hasta el doble de los valores encontrados en este trabajo, incluso en la temporada seca (Ricalde et al., 2010). Esto indica que esta especie es muy eficiente en la procuración y almacenamiento de agua. Aunque los valores de acidez tisular no están dentro de los mayores reportados para las plantas CAM de selvas de Yucatán, su valor de importancia ecológica está entre las primeras 12 especies terrestres perennes en estos ecosistemas (Ricalde et al., 2010). Otras características morfológicas y reproductivas, como la presencia de espinas en los bordes de las hojas y la propagación asexual, permitirán que las poblaciones de Bromelia karatas persistan sin la disminución de su importancia ecológica en el mediano plazo en esta selva baja caducifolia.

\section{Agradecimientos}

Agradecemos a Gabriel Dzib por su apoyo durante el trabajo de campo, a Roberth Us y Luis Simá por proporcionar 
los datos de la estación meteorológica de Dzibilchaltún, y a Manuel Cach, Edilia de la Rosa, Manuela Tamayo, Olivia Hernández y dos revisores anónimos por comentarios a una previa versión del manuscrito. También, agradecemos a las autoridades del Instituto Nacional de Antropología e Historia y a las del Parque Nacional Dzibilchaltún por las facilidades otorgadas para realizar este estudio. C. González-Salvatierra recibió una beca de doctorado por parte del Consejo Nacional de Ciencia y Tecnología, México (CONACYT-172810). Este estudio fue financiado por el Fondo Sectorial SEP-CONACYT 48344/24588 (JLA) y por FOMIX-Yucatán No. 66262 (LMPR).

\section{Literatura citada}

Anderson J.M., Price G.D., Chow W.S., Hope A.B. y Badger M.R. 1997. Reduced levels of cytochrome bf complex in transgenic tobacco leads to marked photochemical reduction of the plastoquinone pool, without significant change in acclimation to irradiance. Photosynthesis Research 53:215-227.

Andrade J.L. 2003. Dew deposition on epiphytic bromeliad leaves: an important event in a Mexican tropical dry deciduous forest. Journal of Tropical Ecology 19:479-488.

Andrade J.L., Cervera J.C. y Graham E.A. 2009. Microenvironments, water relations and productivity of CAM plants. En: de la Barrera E. y Smith W.K. Eds. Perspectives in Biophysical Plant Ecophysiology: A Tribute to Park S. Nobel, pp. 95-120, Universidad Nacional Autónoma de México, México, D.F.

Benzing D.H. 2000. Bromeliaceae: Profile of an Adaptive Radiation. Cambridge University Press, Cambridge.

Buttery B.R., Tan C.S., Buzzell R.I., Gaynor J.D. y MacTavish D.C. 1993. Stomatal numbers of soybean and response to water stress. Plant Soil 149:283-288.

Demmig-Adams B., Adams III W.W., Logan B.A. y Verhoeven A. 1995. Xantophyll cycle-dependent energy dissipation and flexible photosystem II efficiency in plants acclimated to light stress. Australian Journal of Plant Physiology 22:249-260.

Endres L. y Mercier H. 2001. Nitrogen nutrition of bromeliads. En: Horst W.J., Schenk M.K., Bürkert A., Claassen N., Flessa H., Frommer W.B., Goldbach H., Olfs H.W., Römheld V., Sattelmacher B., Schmidhalter U., Schubert S., von Wirén N.V. y Wittenmayer L. Eds. Plant Nutrition. Food Security and Sustainability of Agro-Ecosystems through Basic and Applied Research, pp. 126-127, Kluwer Academic Publishers, Boston.

Fetene M., Lee H.S.J. y Lüttge U. 1990. Photosynthetic acclimation in a terrestrial CAM bromeliad, Bromelia humilis Jacq. New Phytologist 114:399-406.

Givnish T.J. 1988. Adaptation to sun and shade: a whole-plant perspective. Australian Journal of Plant Physiology 15:63-92.

Goldstein G., Andrade J.L. y Nobel P.S. 1991. Differences in water relations parameters for the chlorenchyma and the parenchyma of Opuntia ficus-indica under wet versus dry conditions. Australian Journal of Plant Physiology 18:95-107.

González-Salvatierra C., Andrade J.L., Escalante-Erosa F., GarcíaSosa K. y Peña-Rodríguez L.M. 2010. Antioxidant content in two CAM bromeliad species as a response to seasonal light changes in a tropical dry deciduous forest. Journal of Plant Physiology 167:792-799.
Graham E.A. y Andrade J.L. 2004. Drought tolerance associated with vertical stratification of two co-occurring epiphytic bromeliads in a tropical dry forest. American Journal of Botany 91:699-706.

Griffiths H. 1989. Crassulacean acid metabolism: a re-appraisal of physiological plasticity in form and function. Advances in Botanical Research 15:43-92.

Griffiths H., Lüttge U., Stimmel K.H., Crook C.E., Griffiths N.M. y Smith J.A.C. 1986. Comparative ecophysiology of CAM and $\mathrm{C}_{3}$ bromeliads III. Environmental influences on $\mathrm{CO}_{2}$ assimilation and transpiration. Plant Cell and Environment 9:385-393.

Griffiths H. y Maxwell K. 1999. In memory of C.S. Pittendrigh: Does exposure in forest canopies relate to photoprotective strategies in epiphytic bromeliads? Functional Ecology 13:15-23.

Havaux M., Dall’Osto L. y Bassi R. 2007. Zeaxanthin has enhanced antioxidant capacity with respect to all other xanthophylls in Arabidopsis leaves and functions independent of binding to PSII antennae. Plant Physiology 145:1506-1520.

Hendry G.A.F. y Price A.H. 1993. Stress indicators: chlorophylls and carotenoids. En: Hendry G.A.F. y Grime J.P. Eds. Methods in Comparative Plant Ecology: A Laboratory Manual, pp. 148151, Chapman and Hall, Londres.

Johnson G.N., Young A.J., Scholes J.D. y Horton P. 1993. The dissipation of excess excitation energy in British plant species. Plant, Cell and Environment 16:673-679.

Jones H.G. 1992. Plants and Microclimate: A Quantitative Approach to Environmental Plant Physiology. Cambridge University Press, Cambridge.

Königer M., Harris G.C., Virgo A. y Winter K. 1995. Xanthophyll-cycle pigments and photosynthetic capacity in tropical forest species: a comparative field study on canopy, gap and understory plants. Oecologia 104:280-290.

Larcher W. 2003. Physiological Plant Ecology. Ecophysiology and Stress Physiology of Functional Groups. 4th Edition. Springer, Nueva York.

Logan B., Barker D.H., Demmig-Adams B. y Adams III W.W. 1996. Acclimation of leaf carotenoid composition and ascorbate levels to gradients in the light environment within an Australian rainforest. Plant, Cell and Environment 19:1083-1090.

Lüttge U. 2004. Ecophysiology of crassulacean acid metabolism (CAM). Annals of Botany 93:629-652.

Martin C.E. 1994. Physiological ecology of the Bromeliaceae. The Botanical Review 60:1-82.

Martin C.E., Tüffers A., Herppich W. y von Willert D. 1999. Utilization and dissipation of absorbed light energy in the epiphytic crassulacean acid metabolism bromeliad Tillandsia ionantha. International Journal of Plant Sciences 160:307-313.

Matsubara S., Krause G.H., Aranda J., Virgo A., Beisel K.G., Jahns P. y Winter K. 2009. Sun-shade patterns of leaf carotenoid composition in 86 species of neotropical forest plants. Functional Plant Biology 36:20-36.

Mauseth J.D. 1988. Plant Anatomy. The Benjamin/Cummings Publishing Company Inc., Menlo Park.

Maxwell C., Griffiths H. y Young A.J. 1994. Photosynthetic acclimation to light regime and water stress by the $\mathrm{C}_{3}$-CAM epiphyte Guzmania monostachia: gas-exchange characteristics, photochemical efficiency and the xanthophyll cycle. Functional Ecology 8:746-754.

Maxwell K., Griffiths H., Borland A.M., Young A.J., Broadmeadow M.S.J. y Fordham M.C. 1995. Short-term photosynthetic 
responses of the $\mathrm{C}_{3}$-CAM epiphyte Guzmania monostachia var. monostachia to tropical seasonal transitions under field conditions. Australian Journal of Plant Physiology 22:771-781.

Maxwell K. y Johnson G.N. 2000. Chlorophyll fluorescence - a practical guide. Journal of Experimental Botany 51:659-668.

Mondragón D., Durán R., Ramírez I. y Valverde T. 2004.Temporal variation in the demography of the clonal epiphyte Tillandsia brachycaulos (Bromeliaceae) in the Yucatán Peninsula, México. Journal of Tropical Ecology 20:189-200.

Müller P., Li X.P. y Niyogi K.K. 2001. Non-photochemical quenching. A response to excess light energy. Plant Physiology 125: $1558-1566$.

Niewiadomska E. y Borland A.M. 2008. Crassulacean acid metabolism: a cause or consequence of oxidative stress in plants? En: Lüttge U., Beyschlag W. y Murata J. Eds. Progress in Botany Vol. 69, pp. 247-266, Springer, Berlín.

Niyogi K.K. 2000. Safety valves for photosynthesis. Current Opinion in Plant Biology 3:455-460.

Nobel P.S. 1995. Environmental biology. En: Barbera G., Inglese P. y Pimienta-Barrios E. Eds. Agro-Ecology, Cultivation and Uses of Cactus Pear, pp. 36-57, Plant Production and Protection Paper 132. Publication Division, Food and Agriculture Organization of the United Nations, Roma.

Nobel P.S. y de la Barrera E. 2004. $\mathrm{CO}_{2}$ uptake by the cultivated hemiepiphytic cactus, Hylocereus undatus. Annals of Applied Biology 144:1-8.

Orellana R., Balam M. y Bañuelos I. 1999. Evaluación climática. En: García de Fuentes A., Córdoba y Ordoñez J. y Chico Ponce de León P. Eds. Atlas de Procesos Territoriales de Yucatán, pp. 163-182, Universidad Autónoma de Yucatán, Mérida.

Osborne B.A., Clabby G.T., Horsley D. y Nolan P.F. 1994. Is acclimation required for success in high light environments? A case study using Mycelis muralis (L.) Dumort (Asteraceae). New Phytologist 127:363-375.

Parkhurst D.F. y Mott K.A. 1990. Intercellular diffusion limits to $\mathrm{CO}_{2}$ uptake in leaves: studies in air and helox. Plant Physiology 94:1024-1032.

Parlevliet J., de Haan A.A. y Schellekens J.J.A.M. 1991. Drought Tolerance Research: Possibilities and Constraints. Department of Plant Breeding, Wageningen Agricultural University and Publication Division, Food and Agriculture Organization of the United Nations, Roma.

Patton L. y Jones M.B. 1989. Some relationships between leaf anatomy and photosynthetic characteristics of willows. New Phytologist 111:657-661.

Pearcy R.W., Ehleringer J., Mooney H.A. y Rundel P.W. 1989. Plant Physiological Ecology: Field, Methods and Instrumentation. Chapman and Hall, Nueva York.

Peñuelas J. y Munné-Bosch S. 2005. Isoprenoids: an evolutionary pool for photoprotection. Trends in Plant Science 10:166-169.

Pierce S., Maxwell K., Griffiths H. y Winter K. 2001. Hydrophobic trichome layers and epicuticular wax powders in Bromeliaceae. American Journal of Botany 88:1371-1389.

Pfitsch W.A. y Smith A.P. 1988. Growth and photosynthesis of Aechmea magdalenae, a terrestrial CAM plant in a tropical moist forest, Panama. Journal of Tropical Ecology 4:199-207.

Pimienta-Barrios E., Zañudo-Hernández J. y García-Galindo J. 2006. Fotosíntesis estacional en plantas jóvenes de Agave tequilana. Agrociencia 40:699-709.

Pittendrigh C.S. 1948. The bromeliad-Anopheles-malaria complex in Trinidad. I-The bromeliad flora. Evolution 2:58-89.

Proença S.L. y Sajo M.G. 2007. Anatomia foliar de bromélias ocorrentes em áreas de cerrado do Estado de São Paulo, Brasil. Acta Botanica Brasilica 21:657-673.

Ramírez I.M., Carnevali G. y Chi F. 2004. Guía Ilustrada de las Bromeliaceae de la Porción Mexicana de la Península de Yucatán. Centro de Investigación Científica de Yucatán, A.C., Mérida.

Rascher U., Liebig M. y Lüttge U. 2000. Evaluation of instant light-response curves of chlorophyll fluorescence parameters obtained with a portable chlorophyll fluorometer on site in the field. Plant, Cell and Environment 23:1397-1405.

Reyes-García C. y Griffiths H. 2009. Ecophysiological studies of perennials of the Bromeliaceae family in a dry forest: strategies for survival. En: de la Barrera E. y Smith W.K. Eds. Perspectives in Biophysical Plant Ecophysiology. A Tribute to Park S. Nobel, pp. 121-151, Universidad Nacional Autónoma de México, México, D.F.

Ricalde M.F., Andrade J.L., Duran R., Dupuy J.M., Simá J.L, UsSantamaría R. y Santiago L.S. 2010. Environmental regulation of carbon isotope composition and crassulacean acid metabolism in three plant communities along a water availability gradient. Oecologia 164:871-880.

Ritchie R.J. y Bunthawin S. 2010. The use of pulse amplitude modulation (PAM) fluorometry to measure photosynthesis in a CAM orchid, Dendrobium spp. (D. cv. Viravuth Pink). International Journal of Plant Sciences 171:575-585.

Sajo M.J., Machado S.R. y Carmello-Guerreiro S.M. 1998. Aspectos structurais de folhas de bromélias e suas implicações no agrupamento de espécies. En: Pereira M.V. Ed. Bromélias da Mata Atlântica, pp. 102-111, Salamandra Consultoria Editorial Ltd, Rio de Janeiro.

Scarano F.R., Duarte H.M., Rôças G., Barreto S.M.B., Amado E.F., Reinert F., Wendt T., Mantovani A., Lima H.R.P. y Barros C.F. 2002. Acclimation or stress symptom? An integrated study of intraspecific variation in the clonal plant. Aechmea bromeliifolia, a widespread CAM tank-bromeliad. Botanical Journal of the Linnean Society 140:391-401.

Scatena V.L. y Segecin S. 2005. Anatomia foliar de Tillandsia L. (Bromeliaceae) dos Campos Gerais, Paraná, Brasil. Revista Brasileira de Botânica 28:635-649.

Segecin S. y Scatena V.L. 2004. Anatomia de escapos de Tillandsia L. (Bromeliacea) dos Campos Gerais do Paraná, Brasil. Revista Brasileira de Botânica 27:515-525.

Skillman J.B. y Winter K. 1997. High photosynthetic capacity in a shade-tolerant crassulacean acid metabolism plant: Implications for sunfleck use, nonphotochemical energy dissipation, and susceptibility to photoinhibition. Plant Physiology 113:441-450.

Skillman J.B., Garcia M., Virgo A. y Winter K. 2005. Growth irradiance effects on photosynthesis and growth in two co-occurring shade-tolerant neotropical perennials of contrasting photosynthetic pathways. American Journal of Botany 92:1811-1819.

Smirnoff N. y Crawford R.M.M. 1983. Variation in the structure and response to flooding root aerenchyma in some wetland plants. Annals of Botany 51:237-249.

Smith J.A.C., Griffiths H. y Lüttge U. 1986. Comparative ecophysiology of CAM and $\mathrm{C}_{3}$ bromeliads. I. The ecology of the Bromeliaceae in Trinidad. Plant, Cell and Environment 9:359-376.

Stemke J.A. y Santiago L.S. 2011. Consequences of light absorptance in calculating electron transport rate of desert and succulent 
plants. Photosynthetica 49:195-200

Tanaka Y., Sasaki N. y Ohmiya A. 2008. Biosynthesis of plants pigments: anthocyanins, betalains and carotenoids. The Plant Journal 54:733-749.

Tardy F., Créach A. y Havaux M. 1998. Photosynthetic pigment concentration and interconversions in a pale green Syrian landrace of barley (Hordeum vulgare L., Tadmor) adapted to harsh climatic conditions. Plant, Cell and Environment 21:479-489.

Tyree M.T., Vargas G., Engelbretcht B.M.J. y Kursar T.A. 2002. Drougtht until death do us part: a case study of the desiccation-tolerance of a tropical moist forest seedling-tree, Licania platypus (Hemsl.) Fritsch. Journal of Experimental Botany 53:2239-2247.

Valdez-Hernández M., Andrade J.L., Jackson P.C. y RebolledoVieyra M. 2010. Phenology of five tree species of a tropical dry forest in Yucatan, México: effects of environmental and physiological factors. Plant and Soil 329:155-171.

Van Rensburg L., Kruger G.H.J. y Kruger H. 1994. Assessing the drought-resistance adaptative advantage of some anatomical and physiological features in Nicotiana tabacum. Canadian Journal of Botany 72:1445-1454.

Vogelman T.C., Nishio J.N. y Smith W.K. 1996. Leaves and light capture: light propagation and gradients of carbon fixation within leaves. Trends in Plant Science 1:65-70.

Wellburn A.R. 1994. The spectral determination of chlorophylls $a$ and $b$, as well as total carotenoids, using various solvents with spectrophotometers of different resolution. Journal of Plant Physiology 144:307-313.

Yoder C.K. y Nowak R.S. 1999. Hydraulic lift among native plant species in the Mojave Desert. Plant and Soil 215:93-102.

Zotz G. y Hietz P. 2001. The physiological ecology of vascular epiphytes: current knowledge, open questions. Journal of Experimental Botany 52:2067-2078.

Zotz G. y Andrade J.L. 1998. Water relations of two co-occurring epiphytic bromeliads. Journal of Plant Physiology 152:545$549,552-554$.

Recibido: 21 de mayo de 2012

Aceptado: 3 de agosto de 2012 